PROCEEDINGS OF THE

AMERICAN MATHEMATICAL SOCIETY

Volume 130, Number 7, Pages 1983-1992

S 0002-9939(01)06247-5

Article electronically published on November 21, 2001

\title{
ON THE PREDICTABILITY OF DISCRETE DYNAMICAL SYSTEMS
}

\author{
NILSON C. BERNARDES JR.
}

(Communicated by Alan Dow)

\begin{abstract}
Let $X$ be a metric space. A function $f: X \rightarrow X$ is said to be non-sensitive at a point $a \in X$ if for every $\epsilon>0$ there is a $\delta>0$ such that for any choice of points $a_{0} \in B(a ; \delta), a_{1} \in B\left(f\left(a_{0}\right) ; \delta\right), a_{2} \in B\left(f\left(a_{1}\right) ; \delta\right), \ldots$, we have that $d\left(a_{m}, f^{m}(a)\right)<\epsilon$ for every $m \geq 0$. Let $H(X)$ be the set of all homeomorphisms from $X$ onto $X$ endowed with the topology of uniform convergence. The main goal of the present paper is to prove that for certain spaces $X$, "most" functions in $H(X)$ are non-sensitive at "most" points of $X$.
\end{abstract}

\section{INTRODUCTION}

Consider a discrete dynamical system $(X, f)$, where $X$ is a metric space with metric $d$. If $a \in X$, the sequence $a, f(a), f^{2}(a), \ldots$ can be thought of as the actual behaviour of the system $(X, f)$ at $a$. However, in concrete situations, we are often unable to compute the initial condition $a$ exactly. We just compute a value $a_{0}$ close to $a$. It may also be the case that we cannot compute $f\left(a_{0}\right)$ exactly, but just a value $a_{1}$ close to $f\left(a_{0}\right)$. Then we compute a value $a_{2}$ close to $f\left(a_{1}\right)$ and so on. In this way, we obtain a sequence $a_{0}, a_{1}, a_{2}, \ldots$ that can be thought of as the predicted behaviour of the system $(X, f)$ at $a$. It is natural to ask whether or not this predicted behaviour is close to the actual behaviour of the system. This leads to the following definition:

Definition 1. We say that $f$ is non-sensitive at $a$ if for every $\epsilon>0$ there is a $\delta>0$ such that for any choice of points

$$
a_{0} \in B(a ; \delta), a_{1} \in B\left(f\left(a_{0}\right) ; \delta\right), a_{2} \in B\left(f\left(a_{1}\right) ; \delta\right), \ldots,
$$

we have that

$$
d\left(a_{m}, f^{m}(a)\right)<\epsilon \quad \text { for every } m \geq 0 .
$$

If $f$ is non-sensitive at $a$, then the discrete dynamical system $(X, f)$ is "predictable at $a "$, in the sense that we can predict the future evolution of $a$ in the system forever as accurately as we want provided we can compute the initial condition and the values of $f$ precisely enough.

The above definition may remind the reader of the well-known notion of "shadowing" which, in the case $X$ is compact, may be defined as follows [1]: $f$ is said

Received by the editors August 10, 1999 and, in revised form, January 16, 2001.

2000 Mathematics Subject Classification. Primary 37B25, 37B20, 54H20.

Key words and phrases. Homeomorphisms, predictability, recurrence, Baire category, Lebesgue measure. 
to have the shadowing property (also called the pseudo-orbit tracing property) if for every $\epsilon>0$ there is a $\delta>0$ such that for every sequence $a_{0}, a_{1}, a_{2}, \ldots$ in $X$ satisfying $d\left(f\left(a_{i}\right), a_{i+1}\right)<\delta$ for all $i \geq 0$, there is a point $x \in X$ such that

$$
d\left(a_{m}, f^{m}(x)\right)<\epsilon \text { for all } m \geq 0 .
$$

There is an important difference between "shadowing" and "non-sensitivity" despite the obvious fact that the former is a global notion whereas the latter is a pointwise one. To understand this difference, let $a \in X$ and choose points $a_{0} \in B(a ; \delta)$, $a_{1} \in B\left(f\left(a_{0}\right) ; \delta\right), a_{2} \in B\left(f\left(a_{1}\right) ; \delta\right)$, etc. The shadowing property gives us the existence of a point $x \in X$ (possibly different from $a$ ) such that $d\left(a_{m}, f^{m}(x)\right)<\epsilon$ for all $m \geq 0$. On the other hand, non-sensitivity at $a$ guarantees that this holds with $x=a$. This is a major difference if we are interested in the problem of predictability: the shadowing property guarantees only that the predicted behaviour of the system $(X, f)$ at $a$ is close to some actual behaviour of the system (but this actual behaviour may be different from the actual behaviour at the initial condition $a$ !). For results and further references on "shadowing", see the book [1 by Aoki and Hiraide.

Let $H(X)$ be the set of all homeomorphisms from $X$ onto $X$. If $X$ is compact, we consider $H(X)$ endowed with the supremum metric: $d(f, g)=\sup _{x \in X} d(f(x), g(x))$; $H(X)$ is then a Baire space.

If $M$ is a Baire space, we say that "most elements of $M$ " satisfy a certain property $P$ if the set of all $x \in M$ that do not satisfy property $P$ is of the first category in $M$. The terms "typical" and "generic" are often used instead of "most".

A result related to the notion of predictability was obtained by the author in [2]. Let $B^{n}$ denote the closed unit ball of $\mathbf{R}^{n}$. Then, Theorem 20 of [2] can be stated as follows:

Let $n \geq 2$. For most functions $f \in H\left(B^{n}\right)$, the family $\left\{f^{m} ; m \geq 1\right\}$ is equicontinuous at most points of $B^{n}$.

The equicontinuity of $\left\{f^{m} ; m \geq 1\right\}$ at a point $a$ can be thought of as a kind of "predictability with respect to the initial condition $a$ ". This property is much weaker than the notion of non-sensitivity (which implies that the family $\left\{f^{m} ; m \geq\right.$ $1\}$ is uniformly equicontinuous on the orbit $\left\{a, f(a), f^{2}(a), \ldots\right\}$ of $a$ under $f$ ). Nevertheless, it is indeed true that we can assure the non-sensitivity of $f$ at most points of $B^{n}$, for most $f \in H\left(B^{n}\right)$. In fact, this is true in a much more general setting:

Theorem 2. Fix $n \geq 1$. Let $X$ be a metrizable compact topological n-manifold with (or without) boundary [5] and fix a metric $d$ compatible with the topology of $X$. Then, most functions in $H(X)$ are non-sensitive at most points of $X$.

We shall prove Theorem 2 in Section 4.

In the case $X$ is a metrizable compact smooth manifold without boundary, Odani 6] proved that most functions in $C L D(X)$ (the closure in $H(X)$ of the set of all diffeomorphisms of $X$ ) have the shadowing property. His arguments were based on tools from Differentiable Dynamics. This work was preceeded by Yano [9], who proved that most functions in $H\left(S^{1}\right)$ have the shadowing property (where $S^{1}$ is the unit circle). By Munkres [4] and Whitehead [8], $C L D(X)=H(X)$ whenever $\operatorname{dim} X \leq 3$; but Munkres [4] also showed that this is false if $\operatorname{dim} X>3$. The problem of whether most functions in $H(X)$ have the shadowing property was recently solved in the affirmative for $\operatorname{dim} X$ arbitrary by Pilyugin and Plamenevskaya [7]. However, this problem seems to remain open if $X$ is an arbitrary metrizable compact topological manifold with (or without) boundary. 
An interesting problem is to change the notion of "most" on $B^{n}$ from category sense to measure-theoretic (probability) sense, that is, to consider "most" as meaning "full measure". In this direction we have the following result:

Theorem 3. Fix $n \geq 2$. Most functions in $H\left(B^{n}\right)$ are non-sensitive at every point of a full (Lebesgue) measure subset of $B^{n}$.

As an immediate consequence of this theorem we have the following:

Corollary 4. Let $n \geq 2$. For most functions $f \in H\left(B^{n}\right)$, if $f$ is sensitive to initial conditions on a subset $Y$ of $B^{n}$, then $\bar{Y}$ has Lebesgue measure zero.

Recall that $f$ is sensitive to initial conditions on $Y \subset B^{n}$ if there is an $\epsilon>0$ such that for any $y \in Y$ and any $\delta>0$, there is an $x \in B^{n}$ with $\|x-y\|<\delta$ and there is an $m \geq 1$ so that $\left\|f^{m}(x)-f^{m}(y)\right\| \geq \epsilon$.

We shall prove Theorem 3 in Section 2. Its proof will establish the following result at the same time:

Theorem 5. Let $n \geq 2$. For most functions $f \in H\left(B^{n}\right)$, the set $\Omega_{f}$ of all nonwandering points of $f$ has Lebesgue measure zero.

Theorem 5 improves Theorem 17 of [2], which asserts that for most functions $f \in H\left(B^{n}\right)$, the set $P_{f}$ of all periodic points of $f$ has Lebesgue measure zero. In this direction, it is interesting to observe the following fact:

Proposition 6. Fix $n \geq 2$. For most functions $f \in H\left(B^{n}\right)$, most points of $\Omega_{f}$ are recurrent and non-periodic.

We shall prove Proposition 6 in Section 3.

\section{Proof of Theorems 3 And 5}

Consider $\mathbf{R}^{n}$ endowed with the metric given by its euclidean norm $\|\cdot\|$. Given $A \subset \mathbf{R}^{n}, \bar{A}, \operatorname{Int} A, \operatorname{Bd} A$ and $\operatorname{diam} A$ denote the closure, the interior, the boundary and the diameter of $A$ in $\mathbf{R}^{n}$, respectively, and we define

$$
N_{\delta}(A)=\bigcup_{a \in A}\left\{x \in \mathbf{R}^{n} ;\|x-a\|<\delta\right\} \quad \text { for } \delta>0 .
$$

Throughout the present section, $X$ denotes the closed unit ball of $\mathbf{R}^{n}$ and $\mu$ denotes Lebesgue measure on $\mathbf{R}^{n}$. By an open box we mean a set of the form

$$
\left\{\left(x_{1}, \ldots, x_{n}\right) \in \mathbf{R}^{n} ; \alpha_{i}<x_{i}<\alpha_{i}+\delta \text { for } 1 \leq i \leq n\right\},
$$

where $\alpha_{1}, \ldots, \alpha_{n} \in \mathbf{R}$ and $\delta>0$. A closed box is just the closure of an open box. By a tree we mean a finite rooted tree [3. If $T$ is a tree, $V(T)$ denotes the set of all vertices of $T$. Moreover, if $v_{1}, v_{2} \in V(T)$, we write " $v_{1}>v_{2}$ " or " $v_{2}<v_{1}$ " to mean that $v_{1}$ and $v_{2}$ are adjacent [3] and that the unique path connecting $v_{2}$ to the root of $T$ passes through $v_{1}$. A $B$-tree is a pair $(T, \varphi)$, where $T$ is a tree and $\varphi$ is a bijective correspondence between $V(T)$ and a collection of pairwise disjoint closed boxes contained in $\operatorname{Int} X$. If $(T, \varphi)$ is a $B$-tree, we usually omit the correspondence $\varphi$ and speak just of the $B$-tree $T$; moreover, we make no distinction between a vertex of $T$ and its corresponding closed box.

For each $k \geq 1$, let $A_{k}$ be the set of all functions $f \in H(X)$ for which there are finitely many $B$-trees $T_{1}, \ldots, T_{s}$ so that the following properties are satisfied:

(i) $C \cap F=\emptyset$ whenever $C \in V\left(T_{i}\right), F \in V\left(T_{j}\right)$ and $i \neq j$. 
(ii) $\operatorname{diam} C<1 / k$ whenever $C \in V\left(T_{i}\right)$ for some $1 \leq i \leq s$.

(iii) If $C, F \in V\left(T_{i}\right)$ and $C>F$, then $f(F) \subset \operatorname{Int} C$.

(iv) For each $i$, there is a chain $C_{i, 1}>C_{i, 2}>\cdots>C_{i, t_{i}}$ of successive boxes in $V\left(T_{i}\right)$, beginning with the root $C_{i, 1}$ of $T_{i}$, so that $f\left(C_{i, 1}\right) \subset \operatorname{Int} C_{i, t_{i}}$.

(v) The set

$$
\bigcup\left\{C ; C \in V\left(T_{i}\right)-\left\{C_{i, 1}, \ldots, C_{i, t_{i}}\right\} \text { for some } 1 \leq i \leq s\right\}
$$

together with the set

$$
\bigcup_{i=1}^{s}\left[\left(C_{i, t_{i}}-f\left(C_{i, 1}\right)\right) \cup\left(C_{i, t_{i}-1}-f^{2}\left(C_{i, 1}\right)\right) \cup \ldots \cup\left(C_{i, 1}-f^{t_{i}}\left(C_{i, 1}\right)\right)\right]
$$

form a set of Lebesgue measure $>\mu(X)-1 / k$.

It is immediate to check that each $A_{k}$ is open in $H(X)$. Moreover, $\mu\left(\Omega_{f}\right)<1 / k$ for every $f \in A_{k}$, and so $\mu\left(\Omega_{f}\right)=0$ whenever $f \in \bigcap_{k=1}^{\infty} A_{k}$. Now, suppose $f \in \bigcap_{k=1}^{\infty} A_{k}$. Then, for each $k \geq 1$, there are $B$-trees $T_{k, 1}, \ldots, T_{k, s_{k}}$ so that (i) through (v) hold with $T_{k, 1}, \ldots, T_{k, s_{k}}$ in place of $T_{1}, \ldots, T_{s}$. Put

$$
M_{k}=\bigcup\left\{C ; C \in V\left(T_{k, j}\right) \text { for some } 1 \leq j \leq s_{k}\right\} \quad(k \geq 1) \quad \text { and } \quad M=\bigcap_{r=1}^{\infty} \bigcup_{k=r}^{\infty} M_{k} .
$$

Since $\mu\left(M_{k}\right)>\mu(X)-1 / k$ for every $k \geq 1$, it follows that $\mu(M)=\mu(X)$. Moreover, for each $k \geq 1$, there is a $0<\delta_{k}<1 / k$ such that

$$
f\left(N_{\delta_{k}}(F)\right) \subset \operatorname{Int} C \quad \text { whenever } C>F,
$$

where $C, F \in V\left(T_{k, j}\right)$ for some $1 \leq j \leq s_{k}$, and

$$
f\left(N_{\delta_{k}}\left(C_{k, j, 1}\right)\right) \subset \operatorname{Int} C_{k, j, t_{k, j}},
$$

where $C_{k, j, 1}>\cdots>C_{k, j, t_{k, j}}$ is related to $T_{k, j}\left(1 \leq j \leq s_{k}\right)$ as $C_{i, 1}>\cdots>C_{i, t_{i}}$ is related to $T_{i}$ in property (iv). Hence, if $a \in M_{k}$ and we choose $a_{0} \in B\left(a ; \delta_{k}\right)$, $a_{1} \in B\left(f\left(a_{0}\right) ; \delta_{k}\right), a_{2} \in B\left(f\left(a_{1}\right) ; \delta_{k}\right), \ldots$, then $\left\|a_{m}-f^{m}(a)\right\|<2 / k$ for every $m \geq 0$. This implies that $f$ is non-sensitive at every point of $M$.

It remains to show that each $A_{k}$ is dense in $H(X)$. Fix $k \geq 1, f \in H(X)$ and $\epsilon>0$. Let $0<\delta<1 / k$ be such that

$$
\operatorname{diam} f(Y)<\frac{\epsilon}{2} \quad \text { whenever } Y \subset X \text { and } \operatorname{diam} Y<\delta \text {. }
$$

Let $\mathcal{C}$ be a finite collection of pairwise disjoint closed boxes contained in $\operatorname{Int} X$ such that

$$
\mu\left(X-\bigcup_{C \in \mathcal{C}} C\right)<\frac{1}{k} \text { and } \operatorname{diam} C<\delta \text { for every } C \in \mathcal{C} .
$$

Let $r$ be the cardinality of $\mathcal{C}$ and choose $\eta>0$ such that

$$
\operatorname{diam} f(Y)<\frac{\epsilon}{2^{r+1}} \quad \text { whenever } Y \subset X \text { and } \operatorname{diam} Y<\eta \text {. }
$$

Our next goal is to construct finitely many $B$-trees $T_{1}, \ldots, T_{s}$ satisfying (i) and the following properties:

(a) $\operatorname{diam} C<\delta$ whenever $C \in V\left(T_{i}\right)$ for some $1 \leq i \leq s$.

(b) $\mathcal{C} \subset V\left(T_{1}\right) \cup \ldots \cup V\left(T_{s}\right)$.

(c) If $C, F \in V\left(T_{i}\right)$ and $C>F$, then we have two possibilities:

- either $C \in \mathcal{C}$ and $f(F) \subset C$,

- or $C \notin \mathcal{C}$ and $(\operatorname{Int} C) \cap f(\operatorname{Bd} F) \neq \emptyset$. 
(d) For each $i$, there is a chain $C_{i, 1}>C_{i, 2}>\cdots>C_{i, t_{i}}$ of successive boxes in $V\left(T_{i}\right)$, beginning with the root $C_{i, 1}$ of $T_{i}$, with the following property: $f\left(C_{i, 1}\right) \subset C_{i, t_{i}}$ and/or there is a $C_{i, t_{i}+1} \in V\left(T_{i}\right)$ with $C_{i, t_{i}}>C_{i, t_{i}+1}$ such that $C_{i, 1}, \ldots, C_{i, t_{i}+1} \notin \mathcal{C}$ and both $C_{i, 1}$ and $C_{i, t_{i}+1}$ are contained in an open box $W_{i}$ of diameter $<\eta$.

In order to explain how to construct the trees $T_{1}, \ldots, T_{s}$, we shall need a variable $\mathcal{B}$, which will denote the set of all closed boxes that have already been used in the construction up to the current step (at the beginning we have $\mathcal{B}=\emptyset$ ). It is also important to observe that during the construction the trees will be seen as variables. Even when the construction of a tree $T_{i}$ is apparently done, it may be necessary to change it later on in the process.

We begin by choosing a closed box $C_{1} \in \mathcal{C}$ and by putting it as a vertex of $T_{1}$ (note that now $\mathcal{B}=\left\{C_{1}\right\}$ ). Suppose that in a certain moment $T_{1}$ consists of the vertices $C_{1}<C_{2}<\cdots<C_{j}$. We look at the set $f\left(C_{j}\right)$. There are three possibilities:

Case 1. $f\left(C_{j}\right) \subset C$ for some $C \in \mathcal{B}$.

We stop the construction of $T_{1}$ (for the time being). So, $C_{j}$ is the root of $T_{1}$.

Case 2. $f\left(C_{j}\right) \subset C$ for some $C \in \mathcal{C}-\mathcal{B}$.

Let $C_{j+1}$ be such a $C$. We put $C_{j+1}$ as a vertex of $T_{1}$ adjacent to $C_{j}$ and satisfying $C_{j}<C_{j+1}$.

Case 3. $f\left(C_{j}\right) \not \subset \bigcup\{C ; C \in \mathcal{C}$ or $C \in \mathcal{B}\}$.

Then we also have that $f\left(\operatorname{Bd} C_{j}\right) \not \subset \bigcup\{C ; C \in \mathcal{C}$ or $C \in \mathcal{B}\}$. Hence, we can choose a closed box $C_{j+1} \subset \operatorname{Int} X$ disjoint from each box in $\mathcal{C} \cup \mathcal{B}$ such that $\operatorname{diam} C_{j+1}<\delta$ and $\left(\operatorname{Int} C_{j+1}\right) \cap f\left(\operatorname{Bd} C_{j}\right) \neq \emptyset$. We put $C_{j+1}$ as a vertex of $T_{1}$ adjacent to $C_{j}$ and satisfying $C_{j}<C_{j+1}$.

If Case 1 never happens, then the construction can go on forever. In this case we will stop the construction of $T_{1}$ as soon as we obtain a chain $C_{1,1}>C_{1,2}>\cdots>$ $C_{1, t_{1}}>C_{1, t_{1}+1}$ beginning with the $\operatorname{root} C_{1,1}$ of $T_{1}$ so that $C_{1,1}, \ldots, C_{1, t_{1}+1} \notin \mathcal{C}$ and both $C_{1,1}$ and $C_{1, t_{1}+1}$ are contained in an open box $W_{1}$ of diameter $<\eta$. Since $\mathcal{C}$ is finite and $X$ is bounded, we will obtain such a chain in a finite number of steps.

Suppose that we have already constructed $T_{1}, \ldots, T_{i-1}$. If $\mathcal{B} \supset \mathcal{C}$, we are done. If this is not the case, we choose a $C_{1}^{\prime} \in \mathcal{C}-\mathcal{B}$ and put it as a vertex of $T_{i}$. If in a certain moment $T_{i}$ consists of the vertices $C_{1}^{\prime}<C_{2}^{\prime}<\cdots<C_{j}^{\prime}$, we then look at $f\left(C_{j}^{\prime}\right)$. Cases 2 and 3 are treated as before. However, Case 1 should be divided in two possibilities:

Case 1a. $f\left(C_{j}^{\prime}\right) \subset C$ for some $C \in V\left(T_{i}\right)$.

We stop the construction of $T_{i}$ (for the time being). So, $C_{j}^{\prime}$ is the root of $T_{i}$.

Case 1b. $f\left(C_{j}^{\prime}\right) \subset C$ for some $C \in \mathcal{B}-V\left(T_{i}\right)$.

Let $\tilde{C}$ be such a $C$. Then $\tilde{C}$ is a vertex of a previous tree, say $\tilde{C} \in V\left(T_{i_{0}}\right)$, where $1 \leq i_{0}<i$. In this case we will have no tree $T_{i}$ for the time being. We will just enlarge $T_{i_{0}}$ by putting the chain $C_{1}^{\prime}<C_{2}^{\prime}<\cdots<C_{j}^{\prime}$ as a new branch of it, satisfying the relation $C_{j}^{\prime}<\tilde{C}$. 
If Cases 1a and 1b never happen, we will stop the construction of $T_{i}$ when we obtain a chain $C_{i, 1}>C_{i, 2}>\cdots>C_{i, t_{i}}>C_{i, t_{i}+1}$ beginning with the root $C_{i, 1}$ of $T_{i}$ so that $C_{i, 1}, \ldots, C_{i, t_{i}+1} \notin \mathcal{C}$ and both $C_{i, 1}$ and $C_{i, t_{i}+1}$ are contained in an open box $W_{i}$ of diameter $<\eta$.

By the way the trees $T_{1}, \ldots, T_{s}$ were constructed, it is immediate to check that they have all the desired properties. Note also that $s \leq r$.

Let $I=\left\{i \in\{1, \ldots, s\} ; f\left(C_{i, 1}\right) \subset C_{i, t_{i}}\right\}$ and $J=\{1, \ldots, s\}-I$. For each $C \in V\left(T_{1}\right) \cup \ldots \cup V\left(T_{s}\right)$, choose an open box $V_{C}$ with

$$
\operatorname{diam} V_{C}<\delta \text { and } C \subset V_{C} \subset \overline{V_{C}} \subset \operatorname{Int} X,
$$

so that the family $\left\{\overline{V_{C}}\right\}_{C \in V\left(T_{1}\right) \cup \ldots \cup V\left(T_{s}\right)}$ is pairwise disjoint. We may also assume that

$$
\overline{V_{C_{i, 1}}} \subset W_{i} \text { and } \overline{V_{C_{i, t_{i}+1}}} \subset W_{i} \text { for every } i \in J .
$$

Now, we are going to define a function $g_{0} \in H(X)$ as follows: Suppose that $C, F \in V\left(T_{i}\right)$ for some $i$, and $C>F$. By (c), there is a closed box $B \subset \operatorname{Int} F$ such that $f(B) \subset \operatorname{Int} C$. If $F \notin\left\{C_{i, t_{i}+1} ; i \in J\right\}$, choose a $\varphi \in H\left(\overline{V_{F}}\right)$ such that $\varphi(F) \subset B$ and $\varphi(x)=x$ for all $x \in \operatorname{Bd} V_{F}$, and define

$$
g_{0}(x)=f(\varphi(x)) \quad \text { for all } x \in \overline{V_{F}} .
$$

If $F \in\left\{C_{i, t_{i}+1} ; i \in J\right\}$, then $C \notin \mathcal{C}$ and so $(\operatorname{Int} C) \cap f(\operatorname{Bd} F) \neq \emptyset$. Choose an open box $Z_{F}$ with $F \subset Z_{F} \subset \overline{Z_{F}} \subset V_{F}$ such that there is a closed box $D_{F} \subset V_{F}-\overline{Z_{F}}$ with $f\left(D_{F}\right) \subset \operatorname{Int} C$, choose a $\varphi \in H\left(\overline{Z_{F}}\right)$ such that $\varphi(F) \subset B$ and $\varphi(x)=x$ for all $x \in \operatorname{Bd} Z_{F}$, and define

$$
g_{0}(x)=f(\varphi(x)) \quad \text { for all } x \in \overline{Z_{F}} .
$$

We make this definition for all $C, F \in V\left(T_{i}\right)$ with $C>F(1 \leq i \leq s)$. For $i \in I$, there also exists a closed box $B \subset \operatorname{Int} C_{i, 1}$ such that $f(B) \subset \operatorname{Int} C_{i, t_{i}}$. So, choose a $\varphi \in H\left(\overline{V_{C_{i, 1}}}\right)$ such that $\varphi\left(C_{i, 1}\right) \subset B$ and $\varphi(x)=x$ for all $x \in \operatorname{Bd} V_{C_{i, 1}}$, and put

$$
g_{0}(x)=f(\varphi(x)) \quad \text { for all } x \in \overline{V_{C_{i, 1}}} .
$$

Let $K$ be the union of all these closed boxes where we have already defined $g_{0}$. We finally put $g_{0}(x)=f(x)$ for all $x \in X-K$. Then $g_{0} \in H(X), d\left(g_{0}, f\right)<\epsilon / 2$, and (iii) holds for every $i \in\{1, \ldots, s\}$ and (iv) holds for every $i \in I$ with $g_{0}$ in place of $f$.

Now, we need to change $g_{0}$ a little bit in order to obtain (iv) also for $i \in J$. If $i \in J$ and $1 \leq j \leq t_{i}+1$, we denote the open box $V_{C_{i, j}}$ simply by $V_{i, j}$. Moreover, for $i \in J$, we write $Z_{i}$ and $D_{i}$ in place of $Z_{C_{i, t_{i}+1}}$ and $D_{C_{i, t_{i}+1}}$, respectively. Recall that

$$
D_{i} \subset V_{i, t_{i}+1}-\overline{Z_{i}} \text { and } f\left(D_{i}\right) \subset \operatorname{Int} C_{i, t_{i}} \quad(i \in J) .
$$

Write $J=\left\{i_{1}, \ldots, i_{w}\right\}$ and put

$$
K_{1}=K \cup \overline{V_{i_{2}, 1}} \cup \ldots \cup \overline{V_{i_{w}, 1}} \cup D_{i_{2}} \cup \ldots \cup D_{i_{w}}
$$

Choose

$$
a_{1} \in \operatorname{Int} C_{i_{1}, 1} \subset \overline{V_{i_{1}, 1}} \subset W_{i_{1}}-K_{1} \quad \text { and } \quad b_{1} \in \operatorname{Int} D_{i_{1}} \subset W_{i_{1}}-K_{1} .
$$

Since $K_{1}$ is the union of a finite collection of pairwise disjoint closed boxes contained in $\operatorname{Int} X, W_{i_{1}}-K_{1}$ is connected. So, there is a continuous path $\alpha:[0,1] \rightarrow$ $W_{i_{1}}-K_{1}$ from $a_{1}$ to $b_{1}$. Moreover, we may assume that $\alpha([0,1]) \subset \operatorname{Int} X$. Cover 
$\alpha([0,1])$ by finitely many open balls $B_{1}, \ldots, B_{\ell}$ whose closures are contained in $\left(W_{i_{1}}-K_{1}\right) \cap \operatorname{Int} X$ so that

$$
\overline{B_{1}} \subset \operatorname{Int} C_{i_{1}, 1}, \quad \overline{B_{\ell}} \subset \operatorname{Int} D_{i_{1}} \quad \text { and } \quad B_{i} \cap B_{i+1} \neq \emptyset \text { for every } 1 \leq i<\ell .
$$

By working on $\overline{V_{i_{1}, 1}} \cup \overline{B_{1}} \cup \ldots \cup \overline{B_{\ell}}$, we see that it is possible to construct a $\varphi \in H(X)$ such that

$$
\varphi\left(C_{i_{1}, 1}\right) \subset \operatorname{Int} D_{i_{1}} \quad \text { and } \quad \varphi(x)=x \text { if } x \notin \overline{V_{i_{1}, 1}} \cup \overline{B_{1}} \cup \ldots \cup \overline{B_{\ell}} .
$$

Put $g_{1}=g_{0} \circ \varphi$. Then $g_{1} \in H(X), g_{1}=g_{0}$ on $X-\left(\overline{V_{i_{1}, 1}} \cup \overline{B_{1}} \cup \ldots \cup \overline{B_{\ell}}\right)$ and

$$
g_{1}\left(C_{i_{1}, 1}\right) \subset g_{0}\left(D_{i_{1}}\right)=f\left(D_{i_{1}}\right) \subset \operatorname{Int} C_{i_{1}, t_{i_{1}}} .
$$

Moreover, since $\operatorname{diam}\left(\overline{V_{i_{1}, 1}} \cup \overline{B_{1}} \cup \ldots \cup \overline{B_{\ell}}\right)<\eta$, we have $d\left(g_{1}, g_{0}\right)<\epsilon / 2^{r+1}$ and

$$
\operatorname{diam} g_{1}(Y)<\frac{\epsilon}{2^{r}} \quad \text { whenever } Y \subset X-K \text { and } \operatorname{diam} Y<\eta \text {. }
$$

Put

$$
K_{2}=K \cup \overline{V_{i_{1}, 1}} \cup \overline{V_{i_{3}, 1}} \cup \ldots \cup \overline{V_{i_{w}, 1}} \cup D_{i_{3}} \cup \ldots \cup D_{i_{w}}
$$

Choose

$$
a_{2} \in \operatorname{Int} C_{i_{2}, 1} \subset \overline{V_{i_{2}, 1}} \subset W_{i_{2}}-K_{2} \quad \text { and } \quad b_{2} \in \operatorname{Int} D_{i_{2}} \subset W_{i_{2}}-K_{2},
$$

and argue as before. We then obtain a $g_{2} \in H(X)$ with $g_{2}\left(C_{i_{2}, 1}\right) \subset \operatorname{Int} C_{i_{2}, t_{i_{2}}}$. Moreover, since $g_{2}$ differs from $g_{1}$ only on a set of diameter $<\eta$, we have $d\left(g_{2}, g_{1}\right)<$ $\epsilon / 2^{r}$ and

$$
\operatorname{diam} g_{2}(Y)<\frac{\epsilon}{2^{r-1}} \quad \text { if } Y \subset X-K \text { and } \operatorname{diam} Y<\eta .
$$

By continuing this process, we will obtain a function $g_{w} \in H(X)$ such that

$$
d\left(g_{w}, g_{0}\right)<\frac{\epsilon}{2^{r+1-(w-1)}}+\frac{\epsilon}{2^{r+1-(w-2)}}+\cdots+\frac{\epsilon}{2^{r+1}}<\frac{\epsilon}{2},
$$

and so $d\left(g_{w}, f\right)<\epsilon$. Moreover, properties (iii) and (iv) hold for every $i \in\{1, \ldots, s\}$ if we replace $f$ by $g_{w}$.

Finally, it remains to deal with property (v). Recall that $\mathcal{C} \subset V\left(T_{1}\right) \cup \ldots \cup V\left(T_{s}\right)$ and that $\mu\left(\bigcup_{C \in \mathcal{C}} C\right)>\mu(X)-1 / k$. If we had every box of $\mathcal{C}$ appearing in the first union in property $(\mathrm{v})$ we would be done. However, this may not be the case, since for $i \in I$ there may exist boxes of $\mathcal{C}$ in the chain $C_{i, 1}>C_{i, 2}>\cdots>C_{i, t_{i}}$. For each $i \in I$, let $U_{i}$ be an open box such that

$$
C_{i, 1} \subset U_{i} \subset \overline{U_{i}} \subset V_{C_{i, 1}} \text { and } g_{w}\left(\overline{U_{i}}\right) \subset \operatorname{Int} C_{i, t_{i}} .
$$

Let $\varphi_{i} \in H\left(\overline{U_{i}}\right)$ be such that $\varphi_{i}(x)=x$ for all $x \in \operatorname{Bd} U_{i}$ and $\operatorname{diam} \varphi_{i}\left(C_{i, 1}\right)$ is very small. Put $g=g_{w} \circ \varphi_{i}$ on $\overline{U_{i}}$ for each $i \in I$ and $g=g_{w}$ on $X-\bigcup_{i \in I} \overline{U_{i}}$. Then $g \in H(X)$ and $d(g, f)<\epsilon$, because $g_{w}\left(\overline{U_{i}}\right) \subset f\left(\overline{V_{C_{i, 1}}}\right)$ for each $i \in I$, by the way the $g_{j}$ 's were constructed. Moreover, (iii) and (iv) still hold with $g$ in place of $f$. By choosing $\varphi_{i}$ so that $\operatorname{diam} \varphi_{i}\left(C_{i, 1}\right)$ is small enough, we will have that the measure of

$$
\left(C_{i, t_{i}}-g\left(C_{i, 1}\right)\right) \cup\left(C_{i, t_{i}-1}-g^{2}\left(C_{i, 1}\right)\right) \cup \ldots \cup\left(C_{i, 1}-g^{t_{i}}\left(C_{i, 1}\right)\right)
$$

is so close to the measure of $C_{i, t_{i}} \cup C_{i, t_{i}-1} \cup \ldots \cup C_{i, 1}$ that (v) will also hold with $g$ in place of $f$. This completes the proof. 


\section{Proof of Proposition 6}

Put $X=B^{n}$. We know that for most functions $f \in H(X), \Omega_{f}=\overline{P_{f}}$ and the set of all periodic points of $f$ with period $m$ is dense in the set of all periodic points of $f$ with period $q$ whenever $q$ divides $m$ [2]. Fix an $f \in H(X)$ which has these two properties. Let $R_{f}$ denote the set of all recurrent points of $f$. For each $m \geq 1$ and each $r \geq 1$, let $V_{m, r}=\left\{x \in X ;\left\|x-f^{t}(x)\right\|<1 / m\right.$ for some $\left.t \geq r\right\}$. Then, each $V_{m, r}$ is open and

$$
R_{f}-P_{f}=\bigcap_{m, r, k}\left(V_{m, r}-F_{f^{k}}\right)
$$

(where $F_{f^{k}}$ denotes the set of all fixed points of $f^{k}$ ). Thus, it remains to show that each set $\left(V_{m, r}-F_{f^{k}}\right) \cap \Omega_{f}$ is dense in $\Omega_{f}$. For this purpose, let $U$ be an open set that meets $\Omega_{f}$. Choose a $y \in P_{f} \cap U$. Let $p$ be the period of $y$ and choose an integer $t$ of the form $s p$ (for some $s \geq 1$ ) which is greater than $k$. Now, choose a periodic point $z$ of $f$ with period $t$ which lies in $U$. Then, $z \in\left(V_{m, r}-F_{f^{k}}\right) \cap \Omega_{f} \cap U$, which completes the proof.

\section{Proof of Theorem 2}

Let $i(X)$ denote the interior of the manifold $X$. If $A \subset X$, then $\bar{A}$ and $\operatorname{Int} A$ denote the closure and the interior of $A$ in $X$, respectively, and we define $N_{\delta}(A)$ $=\bigcup_{a \in A} B(a ; \delta)$ for $\delta>0$. Moreover, if $f: X \rightarrow X$ is a mapping, we define $\left(f \circ N_{\delta}\right)^{0}(A)=A,\left(f \circ N_{\delta}\right)^{1}(A)=f\left(N_{\delta}(A)\right),\left(f \circ N_{\delta}\right)^{2}(A)=f\left(N_{\delta}\left(f\left(N_{\delta}(A)\right)\right)\right)$, and so on.

Fix a sequence $z_{1}, z_{2}, \ldots$ in $i(X)$ which is dense in $X$. For each $r \geq 1$ and $k \geq 1$, let $\mathcal{O}_{r, k}$ be the set of all functions $f \in H(X)$ for which there is a closed set $V \subset i(X)$ and there are integers $q \geq 0$ and $m \geq 1$ so that $f^{q}\left(z_{k}\right) \in \operatorname{Int} V, f^{m}(V) \subset \operatorname{Int} V$ and $\operatorname{diam} f^{i}(V)<1 / r$ for $0 \leq i \leq m-1$.

Clearly, each $\mathcal{O}_{r, k}$ is open. Let $f \in \bigcap_{r, k} \mathcal{O}_{r, k}$. By definition, for each $r \geq 1$ and each $k \geq 1$, there are a closed set $V_{r, k} \subset i(X)$ and integers $q_{r, k} \geq 0$ and $m_{r, k} \geq 1$ such that $f^{q_{r, k}}\left(z_{k}\right) \in \operatorname{Int} V_{r, k}, f^{m_{r, k}}\left(V_{r, k}\right) \subset \operatorname{Int} V_{r, k}$ and $\operatorname{diam} f^{i}\left(V_{r, k}\right)<1 / r$ for $0 \leq i \leq m_{r, k}-1$. Let $W_{r, k}$ be an open ball centered at $z_{k}$ such that

$$
f^{q_{r, k}}\left(\overline{W_{r, k}}\right) \subset \operatorname{Int} V_{r, k} \quad \text { and } \quad \operatorname{diam} f^{i}\left(\overline{W_{r, k}}\right)<1 / r \text { for } 0 \leq i \leq q_{r, k}-1 .
$$

Choose $0<\delta_{r, k}<1 / r$ such that

$$
\begin{gathered}
\left(f \circ N_{\delta_{r, k}}\right)^{q_{r, k}}\left(\overline{W_{r, k}}\right) \subset \operatorname{Int} V_{r, k}, \quad\left(f \circ N_{\delta_{r, k}}\right)^{m_{r, k}}\left(V_{r, k}\right) \subset \operatorname{Int} V_{r, k}, \\
\operatorname{diam}\left(f \circ N_{\delta_{r, k}}\right)^{i}\left(\overline{W_{r, k}}\right)<\frac{1}{r}-\delta_{r, k} \quad \text { for } 0 \leq i \leq q_{r, k}-1
\end{gathered}
$$

and

$$
\operatorname{diam}\left(f \circ N_{\delta_{r, k}}\right)^{i}\left(V_{r, k}\right)<\frac{1}{r}-\delta_{r, k} \quad \text { for } 0 \leq i \leq m_{r, k}-1 .
$$

Put $D_{r}=\bigcup_{k=1}^{\infty} W_{r, k}$. Then $D_{r}$ is open and dense in $X$. Moreover, if $a \in D_{r}$, then $a \in W_{r, k}$ for some $k \geq 1$, and so for any choice of points $a_{0} \in B\left(a ; \delta_{r, k}\right)$, $a_{1} \in B\left(f\left(a_{0}\right) ; \delta_{r, k}\right), a_{2} \in B\left(f\left(a_{1}\right) ; \delta_{r, k}\right), \ldots$, we have that $d\left(a_{m}, f^{m}(a)\right)<1 / r$ for every $m \geq 0$. Therefore, $D=\bigcap_{r=1}^{\infty} D_{r}$ is a dense $G_{\delta}$ subset of $X$ and $f$ is nonsensitive at every point of $D$. Thus, it remains to show that each $\mathcal{O}_{r, k}$ is dense in $H(X)$. Fix $r \geq 1, k \geq 1, f \in H(X)$ and $\epsilon>0$. Let $a$ be a cluster point of the 
sequence $\left(f^{j}\left(z_{k}\right)\right)_{j \geq 0}$ and choose a neighborhood $W$ of $a$ in $X$ for which there is a homeomorphism $\psi: W \rightarrow B^{n}$ with

$$
\psi(i(X) \cap \operatorname{Int} W)=\left\{x \in \mathbf{R}^{n} ;\|x\|<1\right\} .
$$

Let $q \geq 0$ be the smallest integer such that $f^{q}\left(z_{k}\right) \in \operatorname{Int} W$. Let $s \geq 1$ be such that $f^{q+s}\left(z_{k}\right) \in \operatorname{Int} W$. We have two possibilities:

Case 1. $n \geq 2$.

We choose a point $b \in(i(X) \cap \operatorname{Int} W)-\left\{f^{j}\left(z_{k}\right) ; j \in \mathbf{Z}\right\}$ so close to $f^{q}\left(z_{k}\right)$ that we have $f^{s}(b) \in \operatorname{Int} W$, and we let $m \geq 1$ be the smallest integer such that $f^{m}(b) \in \operatorname{Int} W$.

Case 2. $n=1$.

We may think of $W$ as being $[-1,1]$. So, we may define the sets

$$
L=\left\{x \in W ;-1<x<f^{q}\left(z_{k}\right)\right\} \quad \text { and } \quad R=\left\{x \in W ; f^{q}\left(z_{k}\right)<x<1\right\} .
$$

If $f^{q+s}\left(z_{k}\right)=f^{q}\left(z_{k}\right)$, choose $b \in R-\left\{f^{j}\left(z_{k}\right) ; j \in \mathbf{Z}\right\}$ so close to $f^{q}\left(z_{k}\right)$ that we have $f^{s}(b)$ and $f^{2 s}(b)$ in Int $W$; then $f^{s}(b)$ or $f^{2 s}(b)$ belong to $R$. If $f^{q+s}\left(z_{k}\right) \neq f^{q}\left(z_{k}\right)$, then either $f^{q+s}\left(z_{k}\right) \in L$ or $f^{q+s}\left(z_{k}\right) \in R$; say $f^{q+s}\left(z_{k}\right) \in R$. In this case, choose $b \in R-\left\{f^{j}\left(z_{k}\right) ; j \in \mathbf{Z}\right\}$ so that $f^{s}(b) \in R$. Let $m \geq 1$ be the smallest integer such that $f^{m}(b) \in R \subset \operatorname{Int} W$.

Let $\phi \in H(X)$ be such that

$$
\phi\left(f^{m}(b)\right)=b \quad \text { and } \quad \phi(x)=x \text { for all } x \in(X-\operatorname{Int} W) \cup\left\{f^{q}\left(z_{k}\right)\right\} .
$$

If $n=1$, we also assume that $\phi(x)=x$ for all $x \in L$. Define $g=\phi \circ f \in H(X)$. Then

$$
g(x)=f(x) \quad \text { whenever } f(x) \in(X-\operatorname{Int} W) \cup\left\{f^{q}\left(z_{k}\right)\right\}
$$

and $b$ is a periodic point of $g$ with period $m$. Moreover,

$$
g^{q}\left(z_{k}\right)=f^{q}\left(z_{k}\right) \in \operatorname{Int} W \quad \text { and } \quad g^{j}\left(z_{k}\right)=f^{j}\left(z_{k}\right) \notin \operatorname{Int} W \text { for } 0 \leq j \leq q-1 .
$$

Let $Z$ and $V$ be closed neighborhoods of $b$ such that

$$
g^{q}\left(z_{k}\right) \in \operatorname{Int} V \subset V \subset \operatorname{Int} Z \subset Z \subset \operatorname{Int} W
$$

and $\psi(Z)$ is a closed ball contained in $\left\{x \in \mathbf{R}^{n} ;\|x\|<1\right\}$. If $n=1$, we also assume that

$$
g(b), \ldots, g^{m-1}(b) \notin Z \text {. }
$$

Since $g(b), \ldots, g^{m-1}(b) \notin Z$, there is a closed neighborhood $V^{\prime}$ of $b$ such that $V^{\prime} \subset \operatorname{Int} Z, Z, g\left(V^{\prime}\right), \ldots, g^{m-1}\left(V^{\prime}\right)$ are pairwise disjoint, $g^{m}\left(V^{\prime}\right) \subset \operatorname{Int} V$ and $\operatorname{diam} g^{i}\left(V^{\prime}\right)<1 / r$ for $1 \leq i \leq m-1$. Now, let $\varphi \in H(X)$ be such that

$$
\varphi(V) \subset V^{\prime} \text { and } \varphi(x)=x \text { for all } x \in(X-\operatorname{Int} Z) \cup\{b\} .
$$

Let $h=g \circ \varphi \in H(X)$. Then

$$
\begin{gathered}
h^{q}\left(z_{k}\right)=g^{q}\left(z_{k}\right) \in \operatorname{Int} V \\
h^{m}(V)=h^{m-1}(g(\varphi(V))) \subset g^{m}\left(V^{\prime}\right) \subset \operatorname{Int} V
\end{gathered}
$$

and

$$
\operatorname{diam} h^{i}(V) \leq \operatorname{diam} g^{i}\left(V^{\prime}\right)<\frac{1}{r} \quad \text { for } 1 \leq i \leq m-1 .
$$

Moreover, by choosing $W$ small enough we can also guarantee that $\operatorname{diam} V<1 / r$ (hence, $\left.h \in \mathcal{O}_{r, k}\right)$ and $d(h, f)<\epsilon$. This completes the proof. 


\section{REFERENCES}

[1] N. Aoki and K. Hiraide, Topological Theory of Dynamical Systems: Recent Advances, NorthHolland, 1994. MR 95m:58095

[2] N. C. Bernardes Jr., On the dynamics of homeomorphisms on the unit ball of $\mathbf{R}^{n}$, Positivity 3 (1999), 149-172. MR 2000g:37018

[3] R. Diestel, Graph Theory, Springer-Verlag, 1997. CMP 97:12

[4] J. R. Munkres, Obstructions to the smoothing of piecewise-differentiable homeomorphisms, Ann. of Math. 72 (1960), 521-554. MR 22:12534

[5] J. R. Munkres, Elements of Algebraic Topology, Addison-Wesley Publishing Company, 1984. MR 85m:55001

[6] K. Odani, Generic homeomorphisms have the pseudo-orbit tracing property, Proc. Amer. Math. Soc. 110 (1990), 281-284. MR 90m:58111

[7] S. Yu. Pilyugin and O. B. Plamenevskaya, Shadowing is generic, Topology Appl. 97 (1999), 253-266. MR 2000i:37023

[8] J. H. C. Whitehead, Manifolds with transverse fields in Euclidean space, Ann. of Math. 73 (1961), 154-212. MR 23a:2225

[9] K. Yano, Generic homeomorphisms of $S^{1}$ have the pseudo orbit tracing property, J. Fac. Sci. Univ. Tokyo Sect. IA Math. 34 (1987), 51-55. MR 88e:58051

Instituto de Matemática, Universidade Federal Fluminense, Rua Mário Santos Braga S/N, 24020-140, Niterói, RJ, BRAsiL

E-mail address: bernardes@mat.uff.br 